\title{
Solving the Location Problem of Printers in a University Campus using $p$-median Location Model and AnyLogic Simulation
}

\author{
Xu Sun ${ }^{1}$, Hao $\mathrm{Yu}^{1}$ and Wei Deng Solvang ${ }^{1}$
}

\begin{abstract}
The location decision on service facilities is of significant importance in determining the accessibility of the service provided. Due to this reason, it has been extensively focused over the past decades by both researchers and practitioners. This paper investigates a novel two-phase hybrid method combining both optimization model and agent-based simulation in order to solve the location problem of printers at a building of UiT The Arctic University of Norway, Narvik campus. In the first phase, the $p$-median location problem is employed to select the optimal locations of printers from a number of pre-determined candidate points so that the total travel distance by both employees and students can be minimized. In the second phase, both the original and the optimal location plans of printers are tested, validated and visualized with the help of AnyLogic simulation package. The result of the case study shows, however, the mathematically optimized solution may not yield a better performance under a realistic environment due to the simplification made and incapability to deal with the randomness. This has revealed that AnyLogic simulation can be used as a powerful tool to validate and visualize the result obtained from an optimization model and to make suggestions on the improvement.
\end{abstract}

Keywords: Location problem, Service facility, Optimization, p-median model, Simulation, AnyLogic

\section{Introduction}

The location problem of printers in a university campus is to select the optimal locations from a set of pre-determined candidates so that the accessibility and satisfaction of users (students and employees in this case) can be improved. Considering the nature of this problem, it is a service location and network design problem that has already been extensively focused on and investigated by both researchers and practitioners for more than half a century. In management science, the basic idea of this problem is to locate a number of facilities and, meanwhile, to allocate customer demand to different

\footnotetext{
${ }^{1} \mathrm{Xu}$ Sun, Hao Yu $(\bowtie)$ and Wei Deng Solvang

Department of Industrial Engineering, Faculty of Engineering Science and Technology, UiT

The Arctic University of Norway, Narvik, Norway

\{xu.sun, hao.yu, wei.d.solvang\}@uit.no
} 
facilities [1]. Over the years, several methods, i.e., mathematical optimization model, analytical hierarchy process (AHP), geographical information system (GIS), etc., have been developed and used to support the decision-making of the location problem and network design of service facility.

Based on a previous research given by Yu et al. [2], this paper presents an improved hybrid method for the location problem of printers in a university campus in Norway. The rest of the paper is structured as follows. Section 2 presents the methodological development. Section 3 illustrates the application of the proposed method with a real world case study at UiT The Arctic University of Norway, Narvik campus. The result and discussion are given in Section 4. Finally, section 5 concludes the paper.

\section{Method}

In order to support the decision-making on service facility location problems, a novel two-phase hybrid method combining both optimization model and agent-based simulation is developed. Fig. 1 illustrates a general framework of the method. First, based on the problem identified, a mathematical optimization model is formulated to make the optimal decisions on both facility location and demand allocation. After that, the result is validated and is visualized in a realistic simulation environment. If the result fulfills the performance required, it will be suggested and be visualized to the decision-makers. Otherwise, all the previous steps need to be re-visited in order to identify the problem of optimization.

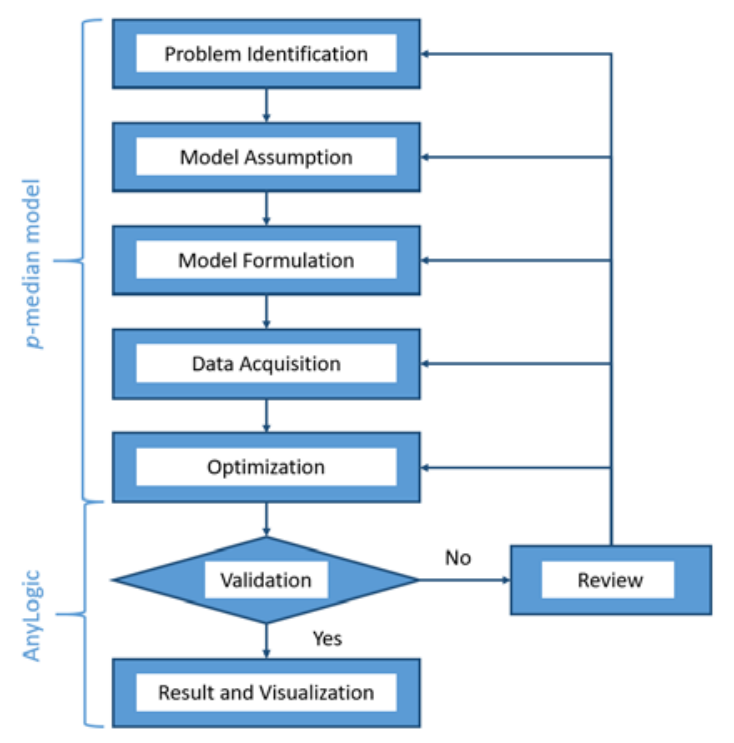

Fig. 1. Framework of the method. 
In this paper, the $p$-median model is employed to make the optimal decisions of the locations of printers. Then, AnyLogic is applied to create a realistic simulation environment and to validate the optimal result obtained.

\section{1 p-median Location Problem}

The $p$-median location problem was first put forward by Hakimi [3]. Since then, it has been extensively investigated and the research focus has been given to both the development of efficient computational algorithms and the application in real-world network design problems. Wheeler [4] investigated a location problem of patrol areas with the help of $p$-median model in order to improve both traveling distance to calls and workload equality. Combining a $p$-median problem with a novel part assignment procedure, Won and Logendran [5] studied the balanced cell formulation in a cellular production process. Incorporating with the environmental evaluation, Pamučar et al. [6] formulated a green $p$-median model for the optimization of city logistics terminals. Taleshian and Fathali [7] proposed a fuzzy $p$-median location problem in order to properly manage the uncertainty in decision-making. Adler at al. [8] investigated a $p$-hub median problem for the network and hub design of air transport in order to deal with the demand expansion in African aviation market. Yu and Solvang [9] employed both maximal covering model and $p$-median model to improve the post office relocation decisions in a city in Norway.

The purpose of the $p$-median model is, through the optimal location-allocation decisions, to minimize the total travel distance in the service network. A mathematical formulation of the $p$-median location problem is given in Eqs. (1-5) [9]. Herein, $I$ and $J$ are the sets of customers and candidate locations for service facility, respectively. The demand from customer $i$ is represented by $q_{i}$, and $d_{i j}$ is the distance between $i$ and $j$. Variable $x_{j}$ determines if a facility is opened or not, and variable $u_{i j}$ determines if the demand from $i$ is served by $j$. Finally, $p$ specifies the number of service facilities in the system.

$$
\begin{aligned}
& \operatorname{Min} \sum_{i \in I} q_{i} d_{i j} u_{i j} \\
& \text { S.t. } \\
& \sum_{j \in J} u_{i j}=1, \forall i \in I \\
& u_{i j} \leq x_{j}, \forall i \in I, j \in J \\
& \sum_{j \in J} x_{j}=p \\
& u_{i j}, x_{j} \in\{0,1\}, \forall i \in I, j \in J
\end{aligned}
$$

Objective function (1) minimizes the total travel distance to satisfy all the customer demands. Constraint (2) assigns each customer to one service facility. Constraint (3) requires a customer can only be allocated to an opened facility. Constraint (4) is the 
requirement on the number of service facilities. Constraint (5) is the binary requirement of decision variables.

\subsection{AnyLogic Simulation}

AnyLogic is a professional simulation software package with a graphical interface, which can be used to create a realistic virtual environment for large and complex systems with different types of behavior (discrete, continuous and hybrid) [10, 11]. AnyLogic is a powerful and flexible tool that enables the modeling of three main features in a simulation: discrete event, agent-based system and system dynamics, namely, which can be combined in order to create a more accurate representation of a complex process or system in the real world.

AnyLogic equips a wide range of built-in modules and database that can easily and quickly be used to create the simulation of a complex system in a great number of industries and service sectors, i.e., manufacturing, logistics and supply chain, networks, dynamic systems, business processes, healthcare, customer behavior, and transportation, etc. Furthermore, for obtaining the analysis and implication from the simulation, AnyLogic has a set of analytical and optimization tools that can be accessed from the modeling environment directly [12].

Except from the standard resources, AnyLogic also enables users to build a highly customized simulation based on the features of the systems modeled. In this regards, Yang et al. [10] investigated the passenger flow at the entrance of a subway station with agent-based pedestrian library in AnyLogic in order to optimize the number of ticket windows opened in peak and off-peak periods. In order to understand the influence of the adoption of electric vehicles on pedestrian traffic safety, Karaaslan et al. [13] built up and studied an agent-based simulation of a real intersection with AnyLogic. Kim et al. [14] used AnyLogic to optimize the location-allocation problem of a biomass supply chain.

\section{$3 \quad$ Case Study}

Combining with both p-median location model and agent-based simulation in AnyLogic, we present a case study of the location problem of printers at the third floor of the main building at UiT The Arctic University of Norway, Narvik campus. The objective is to locate five printers in order to minimize the total travel distance. The optimization process and result with $p$-median model have been given by Yu et al. [2].

In order to simplify the problem, several assumptions are made.

1. Each room is considered as a unique customer demand point and a set of candidate locations is pre-determined.

2. The users are divided into three groups: academic employees, laboratory employees and students. The demand for printing service from different types of users are by no means identical.

3. The demand is aggregated at the center point of each room. 
4. The demand is associated with three influencing factors: type of user, printing frequency and number of user, respectively. Besides, it is also adjusted by the sensitivity to distance from different types of users.

5. The distances between the customer locations and the candidate locations of printers are approximated by the Manhattan distance.

Fig. 2 illustrates the layout of the studied area. The original location plan (red squares) and the optimal location plan (green squares) are also given in the figure.

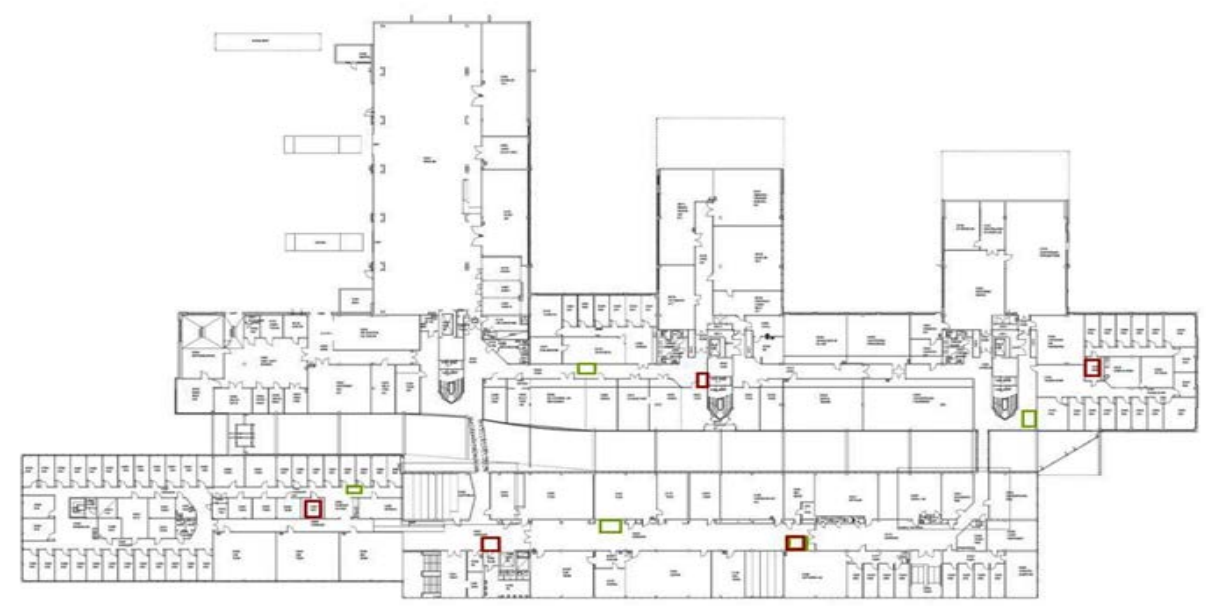

Fig. 2. The building layout and the printer locations in the original plan and the optimized plan.

In this paper, both the original and the optimal location plans of printers are simulated in AnyLogic in order to validate the optimization and visualize the result under a realistic environment. In this modeling and simulation environment, there are many independent objects/individuals (students and employees), so an agent-based approach is used. Compared with the original optimization procedures, several realistic assumption are made as follows in order to have a better representation of the real problem and generate a more reliable analysis.

1. In order to maintain the consistency, the customer demand estimated by Yu et al. [2] is used in the simulation for determining the generation of agents (number and frequency).

2. Instead of aggregating all the customers in the center point of each room, the customer demand can be generated at a random location within the room in the simulation.

3. Instead of using the Manhattan distance to calculate the distances between the customer locations and the candidate locations of printers, the real routes are defined in the simulation

4. Compared the optimization environment from Yu et al. [2] with the current layout of the building, significant changes of the layout of the area served by 
the left most printer in Fig.2 are observed. Thus, this part is not taken into account in the simulation and only the four-printer scenario is tested.

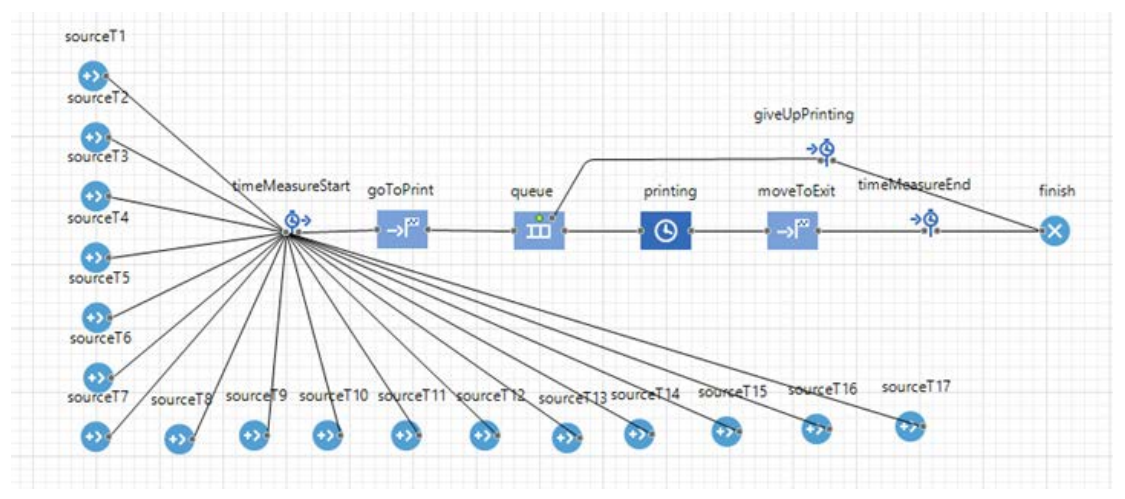

Fig. 3. Agent-based mobile process diagram in AnyLogic.

The flow chart of agent movement is illustrated in Fig.3. The sources (students and employees) are randomly generated from respective classrooms or offices. They will go to the printing area via the real routes defined, use the printers and then move to the exit. In this case, the goal is to calculate the movement distance of all the agents within the studied period. In order to simplify the calculation and reduce the simulation time, we only considered the movement distance in one direction: from the offices or classrooms to the printers. The distance in the reverse direction (back to the rooms) is assumed to be the same as that in the forward direction, so it will not influence the result of the comparison between different location plans. In addition, the movement speed of all the agents is set to $1 \mathrm{~m} / \mathrm{s}$, so the movement distance is directly proportional to the time consumed in the movement.

\section{$4 \quad$ Result and Discussion}

Table 1 illustrates the comparison between the performance evaluation conducted by both optimization method and AnyLogic simulation. It is interesting to observe that, with different approaches, controversial results may be obtained. In this case, the result of the optimization by $p$-median model, which suggests the total travel distance may be reduced by $10 \%$ through relocating the printers, is, however, not supported by the simulation result that shows the original location plan has a better performance.

Table 1 Performance evaluation of the optimal location plan and the original location plan in both optimization and simulation environments

\begin{tabular}{lllll}
\hline Performance evaluation & \multirow{2}{*}{ Optimization } & \multicolumn{2}{l}{ Simulation } \\
\cline { 3 - 5 } & & 1 month & 3 month & 5 month \\
\hline Reduction of total travel distance & $10 \%$ & $-8.06 \%$ & $-7.08 \%$ & $-7.36 \%$ \\
\hline
\end{tabular}


As the procedures given in Fig.1, the mathematical modeling, assumptions and solution code were carefully re-visited in order to identify the problems of the optimization method. In this case study, the main problems of the use of $p$-median location model are the two assumptions made in order to simply the optimization.

1. First, the aggregation of customer demand at the center point neglects the randomness of the real demand generation, which may have a critical impact on the objective value and decision-making especially in a small-scale problem.

2. The most critical problem is the Manhattan distance used in the optimization. The distances between two points is one of the most important influencing factors in the $p$-median location problem, However, as shown in Fig. 4, Manhattan distance cannot always give a realistic representation of the actual distance traveled and thus may lead to an improper result.

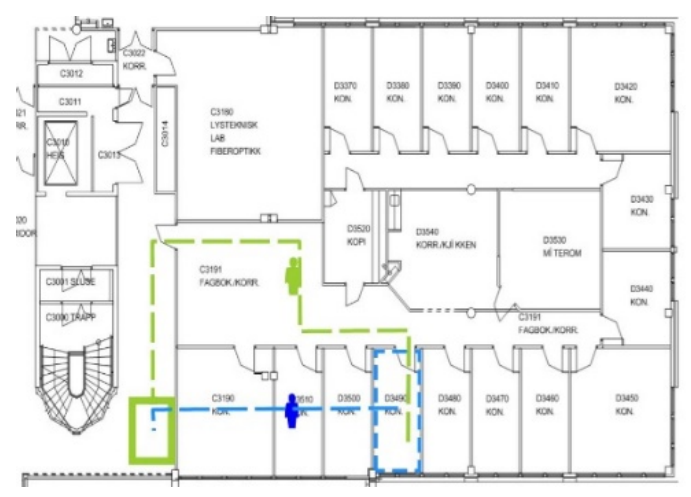

Fig. 4. Illustration of the difference between the Manhattan distance (blue) and the actual distance (green).

In order to solve the aforementioned problems, a stochastic $p$-median model may be formulated so that the randomness of customer generation can be accounted. Moreover, a more realistic distance calculation should be used.

\section{Conclusions}

In this paper, the location problem of printers in a university campus in Norway is investigated using a two-phase hybrid method combining both optimization method and AnyLogic simulation. First, the $p$-median location model was used to optimize the location plan of printers, and then the result was tested in AnyLogic simulation. The simulation result has revealed the problems related to the assumptions and simplifications of the original optimization. The research has proved the effectiveness of AnyLogic simulation in the validation and visualization of the result of optimization. Future research may be conducted in order to address the problems identified in the 
case study. Furthermore, with the help of AnyLogic simulation, not only the movement of agents can be estimated but also comprehensive analysis can be conducted to analyze the overall performance of the printers, i.e., usage of different printers, queueing time, etc.

\section{Reference}

1. Abareshi, M., and Zaferanieh, M.: 'A bi-level capacitated P-median facility location problem with the most likely allocation solution', Transportation Research Part B: Methodological, 2019, 123, pp. 1-20

2. Yu, H., Solvang, W.D., and Yang, J.G.: 'Improving Accessibility and Efficiency of Service Facility through Location-based Approach: A Case Study at Narvik University College', Advanced Material Research, 2014, 1039, pp. 593-602

3. Hakimi, S.L.: 'Optimum distribution of switching centers in a communication network and some related graph theoretic problems', Operations research, 1965, 13, (3), pp. 462-475

4. Wheeler, A.P.: 'Creating optimal patrol areas using the P-median model', Policing: An International Journal, 2019, 42, (3), pp. 318-333

5. Won, Y., and Logendran, R.: 'Effective two-phase p-median approach for the balanced cell formation in the design of cellular manufacturing system', International Journal of Production Research, 2015, 53, (9), pp. 2730-2750

6. Pamučar, D., Vasin, L., Atanasković, P., and Miličić, M.: 'Planning the City Logistics Terminal Location by Applying the Green-Median Model and Type-2 Neurofuzzy Network', Computational intelligence and neuroscience, 2016, Article ID:6792818

7. Taleshian, F., and Fathali, J.: 'A Mathematical Model for Fuzzy-Median Problem with Fuzzy Weights and Variables', Advances in Operations Research, 2016, Article ID:7590492

8. Adler, N., Njoya, E.T., and Volta, N.: 'The multi-airline p-hub median problem applied to the African aviation market’, Transportation Research Part A: Policy and Practice, 2018, 107, pp. 187-202

9. Yu, H., and Solvang, W.D.: 'A Comparison of Two Location Models in Optimizing the Decision-making on the Relocation Problem of Post Offices at Narvik, Norway’, Proceeding of IEEE International Conference on Industrial Engineering and Engineering Management, Thailand, 2018, pp. 814-818

10. Yang, Y., Li, J., and Zhao, Q.: 'Study on passenger flow simulation in urban subway station based on anylogic', Journal of Software, 2014, 9, (1), pp. 140-146

11. Borshchev, A., Karpov, Y., and Kharitonov, V.: 'Distributed simulation of hybrid systems with AnyLogic and HLA', Future Generation Computer Systems, 2002, 18(6), pp. 829-839

12. Karpov, Y.G., Ivanovski, R.I., Voropai, N.I., and Popov, D.B.: 'Hierarchical modeling of electric power system expansion by anylogic simulation software', Proceeding of IEEE Power Tech Conference, Russia, 2015, pp. 1-5

13. Karaaslan, E., Noori, M., Lee, J., Wang, L., Tatari, O., and Abdel-Aty, M.: 'Modeling the effect of electric vehicle adoption on pedestrian traffic safety: An agent-based approach', Transportation Research Part C: Emerging Technologies, 2018, 93, pp. 198-210

14. Kim, S., Kim, S., and Kiniry, J.R.: 'Two-phase simulation-based location-allocation optimization of biomass storage distribution', Simulation Modelling Practice and Theory, 2018, 86, pp. $155-168$ 